\title{
Ensartinib (X-396) a novel drug for anaplastic lymphoma kinase- positive non-small cell lung cancer patients: we need smart trials to avoid wasting good bullets
}

\author{
Gianluca Spitaleri, Antonio Passaro, Filippo de Marinis \\ Division of Thoracic Oncology, IEO, European Institute of Oncology IRCCS, Milan, Italy \\ Correspondence to: Gianluca Spitaleri, MD. Division of Thoracic Oncology, IEO, European Institute of Oncology IRCCS, Via G. Ripamonti, 435- \\ 20141 Milan, Italy. Email: Gianluca.spitaleri@ieo.it. \\ Comment on: Horn L, Infante JR, Reckamp KL, et al. Ensartinib (X-396) in ALK-Positive Non-Small Cell Lung Cancer: Results from a First-in- \\ Human Phase I/II, Multicenter Study. Clin Cancer Res 2018;24:2771-9.
}

Submitted Aug 23, 2018. Accepted for publication Sep 03, 2018.

doi: $10.21037 /$ cco.2018.09.03

View this article at: http://dx.doi.org/10.21037/cco.2018.09.03

Around $3-7 \%$ of patients with non-small cell lung cancer (NSCLC) have a translocation in the gene for anaplastic lymphoma kinase (ALK) (1). Since the first report of ALK detection in NSCLC in 2007, the prognosis and the treatment landscape has been revolutionized for this setting of patients (2). Crizotinib was the first-in-class ALK inhibitor (ALK-i) that demonstrated to be more efficacious than first-line chemotherapy with a median overall survival (OS) still not reached for the patients randomized to crizotinib and a 4-year survival rate of $56.6 \%$ (3). Despite these great results, the majority of the patients develop resistance to crizotinib [the median progression-free survival (mPFS), being less than 11 months], and central nervous system (CNS) is the most common site of disease progression. Moreover, mechanisms of resistance include secondary ALK mutations, amplification of ALK, and activation of bypass signaling networks. Second/third ALK generation drugs have been developed to improve target specificity and activity in the CNS. Alectinib was the first ALK-i to be tested in a head-to-head trial in comparison with crizotinib, attaining dramatic results (the mPFS was 34.8 months) (4). Up to now, several ALK-i (ceritinib, alectinib, brigatinib, lorlatinib) have been experimented in second or further line setting after crizotinib treatment and all proved to be active both globally and in CNS. Ensartinib (X-396) is a novel second-generation ALK-i that holds much clinical promise. Preclinical data have demonstrated increased potency of the drug as compared with crizotinib and other second-generation ALK-i such as alectinib and ceritinib (5). In vitro, it has been demonstrated that ensartinib has activity against few ALK mutations (L1196M and C1156Y) (6). Ensartinib also demonstrated potent antitumor activity in H3122 lung cancer xenografts that harbored the most common EML4-ALK fusion gene variant-1 (7). In the Clinical Cancer Research fournal this year, Leora Horn and colleagues report a phase I/II, multicenter study of ensartinib in patients with advanced ALK-rearranged NSCLC (8). The trial has been conducted in 13 centers around the United States of America. Ensartinib was generally well tolerated, with rash being the most commonly observed toxicity and the recommended dose was chosen (and not reached) as $225 \mathrm{mg}$ based on the frequency of rash observed at $250 \mathrm{mg}$ without improvement in activity. The drug demonstrated good clinical activity in patients who had received a prior ALK-i, those who were ALK-i naive, and those with CNS disease.

Beyond the dose escalation, 60 ALK-positive NSCLC patients were enrolled in the dose expansion. Among these patients: fifteen (25\%) patients were ALK-i naïve, $29(48 \%)$ were treated only with crizotinib, $8(13 \%)$ were progressing after crizotinib and ceritinib and seven (12\%) treated with three ALK-i (10 with crizotinib, ceritinib and alectinib and two with brigatinib after the 2 ALK-i). Table 1 summarizes the results of the phase II part. For the all 60 patients, the rate of response (RR) was $60 \%$, the duration of response (DOR) was 12.8 months and the median-PFS was 9.2 months. Among the 15 ALK-i naive patients the RR was $80 \%$, the DOR and mPFS 
Table 1 Summary of trial results

\begin{tabular}{lcccc}
\hline Setting & N & RR (\%) & DOR (months) & mPFS (months) \\
\hline All & 60 & 60 & 12.8 & 9.2 \\
ALK-i naïve & 15 & 80 & 24.4 & 26.2 \\
$\begin{array}{l}\text { Post-crizotinib } \\
29\end{array}$ & 69 & 7.4 & 9.0 \\
$\begin{array}{l}\text { Post-crizotinib } \\
+\geq 1 \text { ALK-i }\end{array}$ & 16 & 25 & 4.4 & 1.9 \\
\hline
\end{tabular}

$\mathrm{RR}$, rate of response; DOR, duration of response; mPFS, median progression-free survival; ALK-i, ALK inhibitor.

were 24.4 and 26.2 months, respectively. Among the 29 patients treated with crizotinib only, the RR was $69 \%$, the DOR and mPFS were 7.4 and 9.0 months, respectively. Interestingly, among the 16 patients treated with crizotinib and at least another one ALK-i, the RR was $25 \%$, the DOR was 4.4 months (only four patients), and the mPFS 1.9 months. Twenty-nine ( $48 \%)$ patients had brain metastases at baseline, of which twelve patients had not received any prior radiation. Among the 14 patients with CNS target lesions the intra-cranial-RR was of $64.3 \%$ (including seven RR in patients naïve to radiotherapy). Beyond these clinical results, the authors report also a preclinical activity of ensartinib against some ALK mutations (F1174, C1156Y, L1196M, S1206R, and T1151) while it does not seem to be active against G1202R.

Thus, these results, despite attaining in a small phase I/ II trial, confirm that ensartinib has a good activity against ALK-positive NSCLC patients. The drug produces a result in second-line treatment after crizotinib disease progression in terms of mPFS ( 9.2 months) comparable to the other ALK-i and a great result in the ALK-i naïve patients reaching a mPFS of 26.2 months. However, this result must be confirmed in larger controlled trial in the first-line setting with patient accrual ongoing at the time of editorial writing (NCT01625234).

After crizotinib, a series of new drugs have been introduced in the clinical setting: alectinib as the new standard first-line treatment, ceritinib as second-line after crizotinib treatment, brigatinib approved as second-third line ALK-i by US FDA and in Europe available as expanded access program. Beyond these 'old drugs', ensartinib and lorlatinib seem to have an interesting profile of activity. In the ongoing Phase II trial (NCT01970865) enrolling 198 ALK+ heavily pretreated patients, lorlatinib led to rapid deep and durable systemic and intracranial responses (9). Of a total 139 patients in the cohort EXP 4-5 (2 or
3 prior ALK-i), Alectinib produced a systemic RR of $39 \%$ and IC-RR of $47 \%$. Antitumor activity was seen across a range of ALK resistance mutations, including G1202R and G1202del. The current trend is to test these new drugs in the first-line setting (ongoing trials are available for brigatinib, lorlatinib and ensartinib itself), and we think that it is unrealistic that these drugs can perform better than alectinib (mPFS of 34.8 months). While there are few studies, that are exploring the activity of these potent drugs after alectinib save for the phase II trial of brigatinib in patients progressing after next-generation ALK-i (NCT02706626). With this trend, in the next few years we will have many (too much maybe!) approved ALK-i for the first-line setting and few ones be left over the furtherline settings. Since the drugs have different profile of safety, CNS activity (ceritinib and crizotinib have modest intrabrain penetration) and above all different profile of activity against different ALK mutations we strongly recommend testing these drugs in a drug-sequential trial choosing the second-line ALK-i according the ALK mutation profile status at the time of disease progression.

\section{Acknowledgments}

None.

\section{Footnote}

Conflicts of Interest: The authors have no conflicts of interest to declare.

\section{References}

1. Clinical Lung Cancer Genome Project (CLCGP), Network Genomic Medicine (NGM). A genomics-based classification of human lung tumors. Sci Transl Med 2013;5:209ra153.

2. Soda M, Choi YL, Enomoto M, et al. Identification of the transforming EML4-ALK fusion gene in non-small-cell lung cancer. Nature 2007;448:561-6.

3. Solomon BJ, Kim DW, Wu YL, et al. Final Overall Survival Analysis From a Study Comparing First-Line Crizotinib Versus Chemotherapy in ALK-MutationPositive Non-Small-Cell Lung Cancer. J Clin Oncol 2018;36:2251-8.

4. Camidge RD, Peters S, Mok T, et al. Updated efficacy and safety data from the global phase III ALEX study of alectinib (ALC) vs crizotinib (CZ) in untreated advanced 
ALK+ NSCLC. J Clin Oncol 2018;36:abstr 9043.

5. Singhi EK, Horn L. Background and rationale of the eXalt3 trial investigating X-396 in the treatment of ALK+ non-small-cell lung cancer. Future Oncol 2018;14:1781-7.

6. Pall G. The next-generation ALK inhibitors. Curr Opin Oncol 2015;27:118-24.

7. Lovly CM, Heuckmann JM, de Stanchina E, et al. Insights into ALK-driven cancers revealed through development of novel ALK tyrosine kinase inhibitors. Cancer Res

Cite this article as: Spitaleri G, Passaro A, de Marinis F. Ensartinib (X-396) a novel drug for anaplastic lymphoma kinase-positive non-small cell lung cancer patients: we need smart trials to avoid wasting good bullets. Chin Clin Oncol 2019;8(Suppl 1):S1. doi: 10.21037/cco.2018.09.03
2011;71:4920-31.

8. Horn L, Infante JR, Reckamp KL, et al. Ensartinib (X-396) in ALK-Positive Non-Small Cell Lung Cancer: Results from a First-in-Human Phase I/II, Multicenter Study. Clin Cancer Res 2018;24:2771-9.

9. Besse B, Solomon BJ, Felip E, et al. Lorlatinib in patients (Pts) with previously treated ALK+ advanced non-small cell lung cancer (NSCLC): Updated efficacy and safety. J Clin Oncol 2018;36:abstr 9032. 